\title{
Resistivity and Hall effect at high temperatures in $\mathrm{La}_{0.67} \mathrm{Ca}_{0.33} \mathrm{MnO}_{3}$
}

\author{
G. Jakob, W. Westerburg, F. Martin, and H. Adrian \\ Institut für Physik, Johannes Gutenberg-Universität Mainz, D-55099 Mainz, Germany \\ P. J. M. van Bentum and J. A. A. J. Perenboom \\ High Field Magnet Laboratory, University of Nijmegen, 6525 ED Nijmegen, The Netherlands
}

\begin{abstract}
The resistivity tensors of $\mathrm{La}_{0.67} \mathrm{Ca}_{0.33} \mathrm{MnO}_{3}$ films were investigated at temperatures from the Curie temperature up to $600 \mathrm{~K}$ in magnetic fields up to $20 \mathrm{~T}$. The diagonal transport is described by hopping of a small spin polaron cluster of 4 ions to nearest-neighbor spins. A spin-dependent activation energy and a mean field approximation for the magnetization of clustered polaron spins and unclustered $\mathrm{Mn}$ ion spins allowed the description of $\rho(B, T)$ with a minimum of free parameters. The electron-like low field Hall coefficient showed a thermally activated behavior with an activation energy higher than that extracted from diagonal data. (c) 1999 American Institute of Physics. [S0021-8979(99)19408-8]
\end{abstract}

The double-exchange magnetism provides the basic mechanism favoring charge transport in the ferromagnetic regime of doped manganites of type $\mathrm{A}_{x} \mathrm{~B}_{1-x} \mathrm{MnO}_{3}$. The colossal negative magnetoresistivity found close to the Curie temperature $T_{C}$ is attributed to an improved spin order in the presence of magnetic field. Above $T_{C}$ short-range magnetic correlations in form of magnetic polarons are expected to exist and charge transport by magnetic polaron hopping has been proposed. ${ }^{1}$ A temperature dependence of the resistivity $\rho(T)=\rho_{0} T^{\alpha} \exp \left(E_{A} / k_{B} T\right)$ was found in accordance with the Emin-Holstein theory. ${ }^{2}$ The influence of magnetic fields on polaronic transport is difficult to investigate in manganites due to the necessity of applying high fields at high temperatures. Using conventional cryostats one can decrease the $T_{C}$ by doping in order to access this regime in the temperature magnetic field phase diagram. ${ }^{3}$ Here we present experiments on pure $\mathrm{La}_{0.67} \mathrm{Ca}_{0.33} \mathrm{MnO}_{3}$ films with $T_{C} \approx 236 \mathrm{~K}$, close to that of the bulk material.

Microscopic models for activated transport in the presence of strong electron phonon coupling and polaron formation have been solved in mean field approximation by Millis et $a l .{ }^{4}$ and Sheng et al. ${ }^{5}$ However, closed analytical expressions for temperature and magnetic field dependence of electrical transport are not available. Therefore, we correlate our data to the mean field magnetization of small magnetic clusters in a phenomenological model. This gives a quantitative description of $\rho(B, T)$ over a wide range of the phase diagram and allows an estimation of the cluster size.

Preparation and characterization of the sputtered epitaxial films are described elsewhere. ${ }^{6}$ Measurements on a photolithographically patterned Hall bar have been made from 4 $\mathrm{K}$ up to $300 \mathrm{~K}$ in a standard superconducting magnet cryostat. ${ }^{7}$ Above $300 \mathrm{~K}$ an $8 \mathrm{~T}$ superconducting coil and a 20 $\mathrm{T}$ Bitter type magnet system, which both allowed room temperature access to the magnetic field, have been used. For measurements up to $600 \mathrm{~K}$, we placed a small tube furnace into the respective room temperature bores.

A compilation of high temperature resistivity data is shown in Fig. 1. The zero field data show the transition from a metallic, positive temperature coefficient of the resistivity to a thermally activated behavior at high temperatures with a maximum located at $T_{\max }=240 \mathrm{~K}$. In small field magnetization measurements $(B=20 \mathrm{mT})$, the onset of spontaneous magnetization is detected slightly below this temperature. Magnetic fields broaden the resistivity cusp and shift $T_{\max }$ as shown in Fig. 2. In the high temperature regime, the curves in magnetic fields are asymptotic to the zero field curve.

Analyzing $\rho(B=0 \mathrm{~T})$ in the framework of polaron hopping yields an exponent of the temperature dependent prefactor of $\alpha=1$.6. In Fig. 1 all lines are calculated with the value theoretically expected for nonadiabatic polaron hopping $(\alpha=1.5)$. In the nonadiabatic case, there is only a small probability for the polaron to hop during the existence of the excited state. Trial fits with other models such as variable range hopping and nearest neighbor hopping yielded worse fits with strong systematical deviations. However, an acceptable fit was also possible in terms of adiabatic polaron hopping $(\alpha=1) .{ }^{6}$ From the value of $\rho_{0}$ we cross checked that the adiabatic limit is not fulfilled. ${ }^{3}$ The localized carriers are assumed to distort the surrounding lattice and magnetically polarize the manganese atoms in the neighborhood, thereby gaining exchange energy and forming a bound magnetic

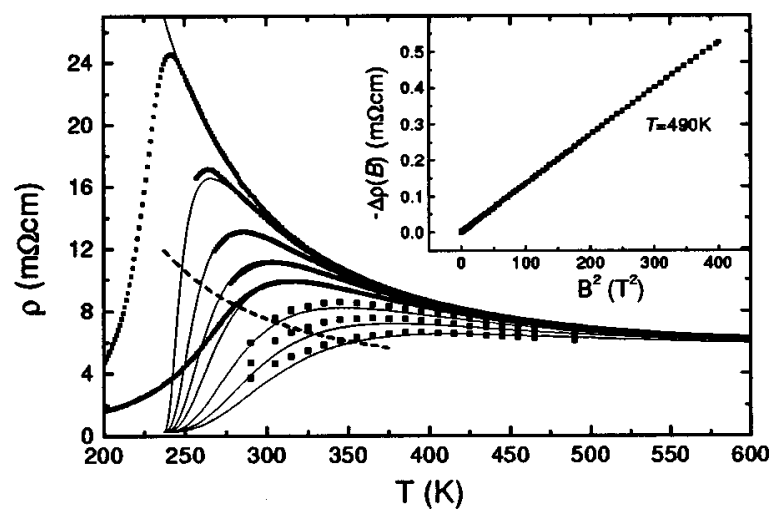

FIG. 1. Temperature dependence of the resistivity in magnetic fields of $B$ $=0,2,4,6,8,12,16,20 \mathrm{~T}$ between 200 and $600 \mathrm{~K}$ (symbols). The data for $B$ $=1,3,5,7,10,14,18 \mathrm{~T}$ are omitted for clarity. The inset shows the $B^{2}$ dependence of the magnetoresistivity for $T=490 \mathrm{~K}$. 


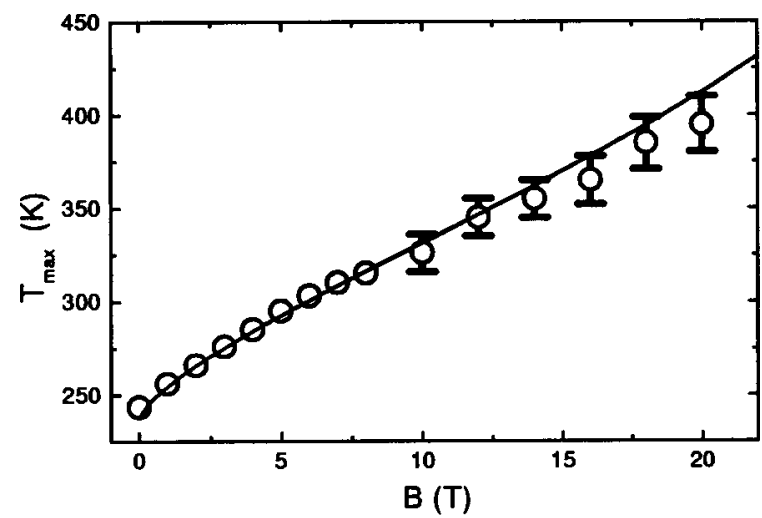

FIG. 2. Measured (symbols) and calculated (line) field dependence of the resistivity maximum.

polaron. ${ }^{8}$ Evidence for the existence of magnetic polarons well above $T_{C}$ has been reported from neutron scattering, ${ }^{9}$ low field magnetization, ${ }^{10,11}$ and electron spin resonance. ${ }^{12}$

For a hopping transport of magnetic polarons, an exchange contribution to the activation energy $E_{A}$ was taken into account by Dionne. ${ }^{13} \mathrm{He}$ expressed $E_{A}$ by a fieldindependent term, arising from electrostatic distortions, and a field-dependent exchange contribution. This model gives a linear dependence between $E_{A}$ and the Brillouin function, i.e., the magnetic field. However, Snyder et al. showed the existence of a $B^{2}$ dependence of the magnetoresistivty $\Delta \rho(B)=\rho(B)-\rho(B=0) .{ }^{14}$ We show that the measured behavior can be described by a simple polaron hopping model based on the following considerations: Since Dionne found an almost purely magnetic polaron with only minor corrections from electrostatic energy, ${ }^{13}$ we consider only the interaction between the spin of an unclustered single ion $\left(N_{I}\right.$ $=1$ ) and the total spin of a ferromagnetic polaron consisting of a cluster of $N_{P}$ ions. The spin alignment due to an applied magnetic field minimizes polaron trapping. In the simplest approximation, the activation energy changes to $E_{A}=E_{A}^{0}(1$ $\left.-\left\langle\cos \Theta_{I P}\right\rangle\right)$. The angle between the single ion spin and the cluster spin, $\Theta_{I P}$, is for uncorrelated spins related to the normalized local magnetizations by $\left\langle\cos \Theta_{I P}\right\rangle=\left\langle\cos \Theta_{I}\right\rangle$ $\times\left\langle\cos \Theta_{P}\right\rangle=\left(\left\langle M_{I}\right\rangle / M_{I}^{S}\right)\left(\left\langle M_{P}\right\rangle / M_{P}^{S}\right)$. We simply calculate in a mean field approximation the magnetizations with the Brillouin functions $B_{J}\left(N_{I}=1, T-T_{C}, B\right)$ and $B_{J}\left(N_{P}, T\right.$ $\left.-T_{C}, B\right)$. As parameters we used a $g$ factor of 2 and the average value of the angular momentum $J=2.28$ per Mn ion. Within this model we can give a closed expression for the resistivity

TABLE I. Fitting parameters according to Eq. (1) for three samples. The values without (with) parenthesis correspond to the nonadiabatic (adiabatic) case.

\begin{tabular}{lccc}
\hline \hline \multicolumn{1}{c}{ Sample } & \multicolumn{1}{c}{$\mathrm{A}$} & $\mathrm{B}$ & $\mathrm{C}$ \\
\hline$\rho_{0}\left(10^{-10} \Omega \mathrm{m} / \mathrm{K}^{\alpha}\right)$ & $6.44(209.7)$ & $6.53(211.4)$ & $4.89(157.2)$ \\
$E_{A}^{0}(\mathrm{meV})$ & $120(103)$ & $96(80)$ & $133(117)$ \\
$T_{C}(\mathrm{~K})$ & $213.3(203.6)$ & $236.4(235.9)$ & $202.0(195.6)$ \\
$N_{P}$ & $3.9(5.9)$ & $3.8(5.0)$ & $5.5(7.8)$ \\
\hline \hline
\end{tabular}

$$
\rho(T, B)=\rho_{0} T^{\alpha} \exp \left(\frac{E_{A}^{0}}{k_{B} T}\left[1-B_{J}\left(N_{I}\right) B_{J}\left(N_{P}\right)\right]\right) .
$$

The fit of the nonadiabatic polaron hopping transport $(\alpha$ $=1.5$ ) to the zero field data fixes the parameters $\rho_{0}$ and $E_{A}^{0}$. In Fig. 1 we fitted the data for $B=4 \mathrm{~T}$ in order to determine the two free parameters $N_{P}=3.8$ and $T_{C}=236 \mathrm{~K}$. The smallness of the cluster size $N_{P}$ limits the spin polarization to the nearest neighborhood. Within our model the above equation describes all other field curves without further free parameters. The field and temperature dependence is implicit in the Brillouin functions. The result of the analysis is shown by lines in Fig. 1, and shows good correspondence between the experimental data (symbols) and the respective calculated curves (lines). Fits with fixed cluster sizes, $N_{P}=1$ and $N_{P}$ $=7$, respectively, yielded significantly less good correspondence. Here, the first value represents a model of pure electron hopping with spin-dependent barrier energies. The numerical value for $N_{P}$ should not be considered as exact, however, it demonstrates that a small finite cluster size is necessary to describe the experimental data.

At temperatures well above the Curie temperature ( $T$ $>2 T_{C}$ ) the Brillouin functions in Eq. (1) reduce to effective susceptibilities and the small correction of the activation energy due to the magnetic field will be quadratic in $B$. Therefore, a Taylor expansion of the exponential will also be quadratic in the leading field-dependent term and one expects: $\Delta \rho(B)=\rho(B)-\rho(B=0) \propto B^{2}$ as it is visible in the inset of Fig. 1.

In Fig. 2 we show the nonlinear shift of $T_{\max }$ with applied magnetic field. In the superconducting magnet system $(B \leqslant 8 \mathrm{~T})$, the data were taken densely in temperature and the error of $T_{\max }$ is smaller than symbol size. In the Bitter magnet system, data spacing is less dense resulting in larger errors. With the parameters fixed from above we can solve Eq. (1) numerically for $T_{\max }(B)$. The result of this inversion is shown as solid line in Fig. 2.

Looking more closely to Fig. 1 deviations are obvious. First, the zero-field fit curve exhibits no maximum but diverges in the low temperature limit. This is because fluctuations are not taken into account and therefore the calculated magnetization is exactly zero above $T_{C}$. Second the calculated resistivity for all magnetic fields approaches a constant value near $T_{C}$. This is an artefact of the model which calculates thermally activated hopping even close to $T_{C}$, where the activation energy vanishes. Our model assumes clusters of $\mathrm{Mn}$ ions surrounded by a paramagnetic background of single $\mathrm{Mn}$ ions. However, close to $T_{C}$ the clusters are all well aligned and also spin ordering of the not clustered $\mathrm{Mn}$ ions is considerably. Thus there is a high probability of having a cluster of $N_{P} \mathrm{Mn}$ ions spin aligned with a neighboring $\mathrm{Mn}$ ion. In our model, this is indistinguishable from a cluster of $N_{P}+1 \mathrm{Mn}$ ions. A growing cluster size near $T_{C}$ was experimentally observed by neutron scattering and magnetization measurements ${ }^{9,11}$ and should be implemented in Eq. (1). A cluster growth would lead to a faster decrease of the resistivity when approaching $T_{C}$ from above than that sketched in Fig. 1. On the other hand, the neglected lattice energy of the polaron will act in the opposite direction. Incorporating these 


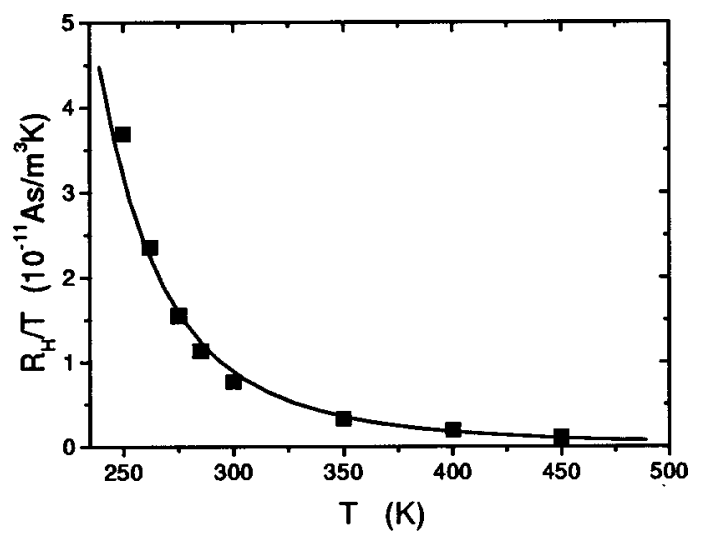

FIG. 3. Hall coefficient $R_{H} / T$ as function of temperature (symbols) and temperature dependence calculated for an activation energy of $165 \mathrm{meV}$ (line).

effects would require a model for cluster growth and additional fitting parameters. Instead we give an estimate of the region where we think Eq. (1), which deals with independent, not aligned clusters and ions, is applicable. As a criterion we choose $\left\langle\Theta_{I P}(B, T)\right\rangle-\left\langle\Theta_{I P}(B=0, T)\right\rangle<10^{\circ}$. The dashed curve in Fig. 1 marks this criterion. Data values above this crossover line correspond to a spin alignment between clusters and single spins deviating less than $10^{\circ}$ from the equilibrium value of $90^{\circ}$ expected for random spin orientations.

In Table I we list the resulting fitting parameters for three investigated samples for the adiabatic $(\alpha=1)$ and nonadiabatic $(\alpha=1.5)$ case, respectively. Regardless of the type of polaron hopping only a finite, small cluster size could successfully describe the experiment.

For the motion of a polaron on a triangular lattice, Friedman and Holstein ${ }^{15}$ found a thermally activated electron-like Hall effect $R_{H} \propto T \exp \left(2 E_{A} / 8 k_{B} T\right)$ from quantum interference between direct and indirect jumps. We find an electron-like linear slope of the Hall voltage in the polaronic regime which changes to a positive slope in the ferromagnetic metallic regime. The evaluation of the Hall effect in the ferro- magnetic state is published elsewhere. ${ }^{7}$ In Fig. 3 we show the Hall coefficient $R_{H} / T$ as function of temperature. From the thermally activated behavior, we can extract an activation energy for the Hall mobility of $(165 \mathrm{meV})$. This value is higher than the activation energy of the drift mobility (96 $\mathrm{meV}$ ), in contradiction to Friedman-Holstein theory and the experimental results found by Jaime $e t$ al. $^{3}$ However, a more general theory of Schnakenberg ${ }^{16}$ found the same activation energy for longitudinal resistivity and Hall mobility.

In summary, we performed detailed high field transport measurements covering a wide temperature and magnetic field range on the colossal magnetoresistive compound $\mathrm{La}_{0.67} \mathrm{Ca}_{0.33} \mathrm{MnO}_{3}$. With a small polaron hopping model we could quantitatively describe $\rho(B, T)$ with a minimum of free parameters. The cluster sizes determined from the fits were in all cases small, containing 4-6 ions only. Also a thermally activated polaronic Hall effect was found, but it did not agree quantitatively with theoretical considerations.

This work was supported by the Deutsche Forschungsgemeinschaft through Project No. JA821/1 and the European Union "TMR-Access to Large Scale Facilities Plan."

${ }^{1}$ D. C. Worledge et al., J. Appl. Phys. 80, 1 (1996); D. C. Worledge, L. Miéville, and T. H. Geballe, Phys. Rev. B 57, 15627 (1998).

${ }^{2}$ D. Emin and T. Holstein, Ann. Phys. (N.Y.) 53, 439 (1969).

${ }^{3}$ M. Jaime et al., Phys. Rev. Lett. 78, 951 (1997).

${ }^{4}$ A. J. Millis, B. I. Shraiman, and R. Mueller, Phys. Rev. Lett. 77, 175 (1996).

${ }^{5}$ L. Sheng, D. Y. Xing, D. N. Sheng, and C. S. Ting, Phys. Rev. Lett. 79, 1710 (1997).

${ }^{6}$ G. Jakob, F. Martin, W. Westerburg, and H. Adrian, J. Magn. Magn. Mater. 177-181, 1247 (1998).

${ }^{7}$ G. Jakob, F. Martin, W. Westerburg, and H. Adrian, Phys. Rev. B 57, 10252 (1998).

${ }^{8}$ P. G. de Gennes, Phys. Rev. 118, 141 (1960).

${ }^{9}$ J. M. De Teresa et al., Nature (London) 386, 256 (1997).

${ }^{10}$ J. Z. Sun et al., Appl. Phys. Lett. 69, 1002 (1996).

${ }^{11}$ V. S. Amaral et al., J. Appl. Phys. 83, 7154 (1998).

${ }^{12}$ M. T. Causa et al., Phys. Rev. B 58, 3233 (1998).

${ }^{13}$ G. F. Dionne, J. Appl. Phys. 79, 5172 (1996).

${ }^{14}$ G. J. Snyder et al., Appl. Phys. Lett. 69, 4254 (1996).

${ }^{15}$ L. Friedman and T. Holstein, Ann. Phys. (N.Y.) 21, 494 (1963).

${ }^{16}$ J. Schnakenberg, Z. Phys. 185, 123 (1965). 